\title{
Preparation and characterization of uniform pseudocubic hematite particles by utilizing polyethylene oxide polymers in forced hydrolysis reaction
}

\author{
Kazuhiko KANDORI ${ }^{\dagger}$ and Gohki YONEKAWA \\ School of Chemistry, Osaka University of Education, 4-698-1 Asahigaoka, Kashiwara, Osaka 582-8582, Japan
}

\begin{abstract}
The monodispersity of pseudocubic hematite particles, produced from a forced hydrolysis reaction of acidic $\mathrm{FeCl}_{3}$ solution, were controlled by using five kinds of polyethylene oxides (PEO; Alkox ${ }^{\circledR}$; Mesei Chemical Works. Ltd.) (0-2 wt.\%). Alkox ${ }^{\circledR}$ possesses nominal formula of $-\left(\mathrm{CH}_{2}-\mathrm{CH}_{2}-\mathrm{O}\right)_{n}-$. The fairly uniform pseudocubic particles was precipitated for PEO with low $\mathrm{M}_{\mathrm{w}}$ value and narrow molecular weight distribution. However, POEs with large molecular weight and wide molecular weight distribution produced polydispersed pseudocubic hematite particles. The PEO with the largest molecular weight attained nearly spherical with large particle size distribution. From the calculation of crystallite size, all the pseudocubic hematite particles were regarded as a polycrystal as well as the large spherical hematite particles produced without Alkox ${ }^{\circledR}$ (control system). This polycrystallinity of the particles provided an evidence that the particles are grown by aggregation of polynuclear (PN) primary particles. The specific surface areas of the particles (SSA) were ranged in $4-10 \mathrm{~m}^{2} / \mathrm{g}$ and no large difference can be recognized among five kinds of PEOs. Since the measured SSA values were corresponded to the SSA one calculated by assuming monodispersed cubic particle with $300 \mathrm{~nm}$ in edge length, the pseudocubic hematite particles are non-porous. The time resolved XRD, TEM, ICP-AES and TOC measurements employed on the systems produced for pseudocubic particles elucidated that the pseudocubic crystal habit was formed by the specific adsorption of PEO molecules together with chloride ions and/or chloro ferric complexes to the \{012\} faces, restraining the growth process through stacking of ultrafine PN particles in the direction of normal to the $\{012\}$ faces but strictly restricting the growth and mutual fusion of PN ones. The uniform pesudocubic particles found to be effective photocatalytic material than spherical and/or polydispersed pseudocubic hematite ones due to their large size with uniform flat crystal faces.
\end{abstract}

(C2014 The Ceramic Society of Japan. All rights reserved.

Key-words : Hematite, Psuedocubic, Particles, Polyethylene oxide, Photocatalysis

[Received May 8, 2014; Accepted June 24, 2014]

\section{Introduction}

Recently design and synthesis of the morphology-controlled colloidal nanoparticles have been intensively investigated for their size and shape-dependent properties. ${ }^{1)-4)}$ Hematite $\left(\alpha-\mathrm{Fe}_{2} \mathrm{O}_{3}\right)$ represents the most important ore of iron and is the most stable iron oxide under ambient conditions. It has been profoundly investigated, therefore, due to its environmental safety and potential applications on gas sensor, catalysis and magnetic storage devices. ${ }^{5)}$ Nowadays, various well-defined simple shapes of hematite particles, such as rods, wire, belt, tubes, ellipsoids, rings and spindles, has been obtained by vapor-phase processes and solid-based wet method such as a forced hydrolysis method. ${ }^{6)-13)}$ Our research group has been reported on the formation of spherical hematite particles by the forced hydrolysis reaction under $100^{\circ} \mathrm{C}$. The authors revealed that the spherical hematite particles are formed by aggregation of fine polynuclear (PN) primary particles produced after dissolved $\beta$ - $\mathrm{FeOOH}$ particles, which appeared at the initial stage of the reaction. ${ }^{14)}$ Similar aggregation mechanism was also reported on the formation of micrometer-sized cubic hematite particles. ${ }^{15)}$

In the past decade, our research group has extended the investigation on the formation of hematite particles from the

Corresponding author: K. Kandori; E-mail: kandori@cc.osakakyoiku.ac.jp forced hydrolysis reaction of acidic $\mathrm{FeCl}_{3}$ solution in the presence of polymers. ${ }^{16-18)}$ As using vinyl series polymers (polyacrylamide, PAAm and polyacrylic acid, PAAc) with a high complexity to $\mathrm{Fe}(\mathrm{III})$ ions and a high adsorption affinity on both $\beta$-FeOOH of initial hydrolysis products of $\mathrm{FeCl}_{3}$ aqueous solution and PN particles, the authors found that these polymers strongly influence on morphology and structure of hematite particles even at extremely low concentrations (less than $4 \times 10^{-3}$ wt.\%). ${ }^{18)}$ The shape of the particles produced with PAAc was ellipsoidal though it was spherical for those formed with PAAm. These findings reveals that the particle shape strongly depends on the adsorption mode and strength of polymers onto $\beta$-FeOOH, PN primary and growing hematite particles regardless of the molecular weight of additives. Based on these results, we investigated further the effects of diblock co-polymer with terminal amino groups, $\left[\mathrm{CH}_{3}-(\mathrm{PEO})_{x}-(\mathrm{PPO})_{y}-\mathrm{NH}_{2}\right]$, so called as Surfonamine $\left.{ }^{\circledR} .19\right)$ Surfonamine with the highest total amine content (PEO contents were over $80 \mathrm{~mol} \%$ :) gave spherical but those with lower total amine contents (PEO contents less than $76 \%$ ) produced pseudocubic hematite particles. However, the uniformity in size of pseudocubic particles was low due to their low molecular weight of Surfonamine employed (600-3000).

It is well known that the growth and/or inhibition of particular crystal faces of the particles usually attain anisotropic particles such as cubic and rod-like shapes. To develop uniform cubic hematite particles, PEO polymers with large molecular weight (larger than $1 \times 10^{5}$ ) are desired because there are large numbers 
of hydrophilic PEO units of those induce a strong adsorption onto $\beta$-FeOOH, PN primary and growing hematite particles. Since the orientation of the uniform cubic particles is ready to take place on the matrix, it is expected to develop new nano-composites. The aim of the present study is to disclose this point. The present study will describe the fundamental information for producing uniform pseudocubic hematite particles and their physicochemical properties. The application of these as-prepared uniform pseudocubic hematite particles was also demonstrated on a photocatalysis reaction.

\section{Experimental procedure}

\subsection{Synthesis of hematite particles}

Hematite particles were prepared from the forced hydrolysis of mixed solution of $\mathrm{FeCl}_{3}$ and $\mathrm{HCl}$ dissolving various amounts of PEO (0-2wt.\%). This procedure was almost the same as employed in our previous paper. ${ }^{19)}$ The total concentrations of $\mathrm{FeCl}_{3}$ and $\mathrm{HCl}$ in a $30 \mathrm{~mL}$ solution were fixed at $3.12 \times 10^{-2}$ and $9.60 \times 10^{-3} \mathrm{~mol} \mathrm{dm}^{-3}$, respectively. This condition without PEO provides fairy uniform spherical particles of ca. $600 \mathrm{~nm}$ in diameter. ${ }^{16-18)}$ Pyrex glass vial containing each $30 \mathrm{~mL}$ of reacting solution was tightly closed with a Teflon-lined screw cap and stood in a conventional forced-air oven at $100^{\circ} \mathrm{C}$ for $7 \mathrm{~d}$. Five kinds of PEO [Alkox ${ }^{\circledR} ;-\left(\mathrm{CH}_{2}-\mathrm{CH}_{2}-\mathrm{O}\right)_{n}$ ], kindly supplied from Mesei Chemical Works. Ltd. (Kyoto, Japan), as listed in Table 1 were used in this study. The number $\left(\mathrm{M}_{\mathrm{n}}\right)$-, weight $\left(\mathrm{M}_{\mathrm{w}}\right)$-average molecular weights and their ratio $\left(\mathrm{M}_{\mathrm{w}} / \mathrm{M}_{\mathrm{n}}\right)$ were listed in Table 1. The molecular weight distribution curves of PEOs are shown in Fig. 1. All the PEOs possess larger $M_{w}$ values, larger than $1 \times 10^{5}$ and its order can be noted as follows, L-11< $\mathrm{R}-150<\mathrm{R}-400<\mathrm{R}-1000 \ll \mathrm{E}-60$. The L-11 has a narrow and sharp distribution curve, suggesting that the number of PEO units

Table 1. Properties of polyethylene oxides $\left(\right.$ Alkox $^{\circledR a}$ a) employed

\begin{tabular}{lccc}
\hline Alkox ${ }^{\circledR}$ & $\begin{array}{c}\text { Number average } \\
\text { molecular weight } \\
\left(\mathrm{M}_{\mathrm{n}}\right)\end{array}$ & $\begin{array}{c}\text { Weight average } \\
\text { molecular weight } \\
\left(\mathrm{M}_{\mathrm{w}}\right)\end{array}$ & $\mathrm{M}_{\mathrm{w}} / \mathrm{M}_{\mathrm{n}}$ \\
\hline L-11 & $7.60 \times 10^{4}$ & $11.6 \times 10^{4}$ & 1.53 \\
R-150 & $2.41 \times 10^{4}$ & $14.4 \times 10^{4}$ & 5.98 \\
R-400 & $2.98 \times 10^{4}$ & $20.2 \times 10^{4}$ & 6.78 \\
R-1000 & $5.70 \times 10^{4}$ & $29.6 \times 10^{4}$ & 5.19 \\
E-60 & $1.97 \times 10^{5}$ & $10.0 \times 10^{5}$ & 5.10 \\
\hline
\end{tabular}

a) Alkox $^{\circledR}$ from Meisei Chemical Works Ltd. (Kyoto, Japan)

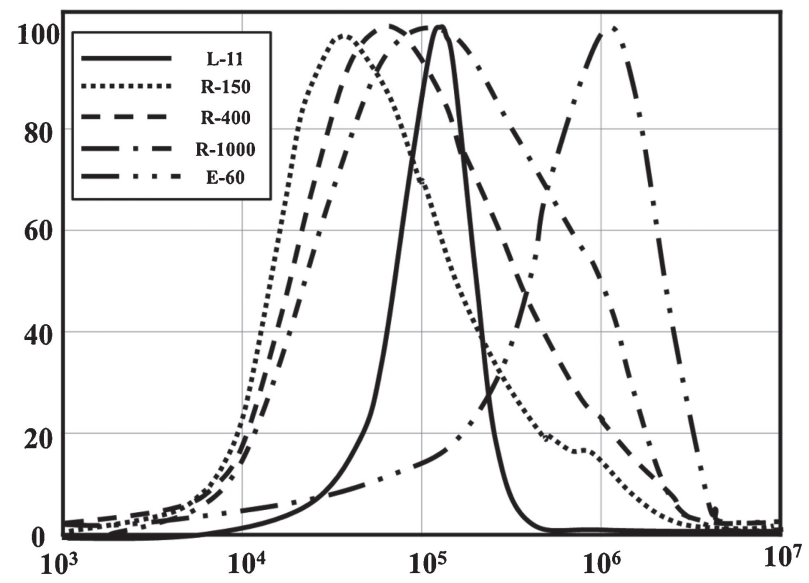

Fig. 1. Molecular weight distribution curves of polyethylene oxides employed measured by GPC method. is considerably ordered. On the other hand, molecular weight distribution curves of the rest of L-11 are widely spread out from $1 \times 10^{3}-10^{7}$. Since the E-60 has the highest $\mathrm{M}_{\mathrm{w}}, 10$ times larger than L-11, the strong effect of E-60 onto particle formation can be presumed. The resulting particles were thoroughly washed by filtration with ultrapure water using $0.2 \mu \mathrm{m}$ Millipore filter and finally dried in air-oven at $40^{\circ} \mathrm{C}$ for $18 \mathrm{~h}$. The solution $\mathrm{pH}$ before and after aging was monitored at room temperature. Guaranteed reagent grade chemicals from Wako Pure Chemical Co. Inc. (Osaka, Japan) were used without further purification. Ultrapure water from Elix (Millipore) were used for the preparation and washing of all particles. Fresh ultrapure water had conductivities ca. $0.06 \mu \mathrm{S} / \mathrm{cm}$.

\subsection{Characterization of the samples}

The morphology and crystal structure of particles were investigated by transmission electron microscope (TEM; JEOL JEM2100), simultaneous thermogravimetry and differential thermal analysis (TG-DTA; SII-7200), Fourier-transform infrared spectrometer (FTIR; Nicolet Protégé 460) embedded in $\mathrm{KBr}$ pellets $(0.2 \mathrm{wt} . \%)$ and X-ray powder diffraction (XRD; Rigaku Rad-RC, $40 \mathrm{kV}, 120 \mathrm{~mA}$ ) techniques. The concentrations of PEOs and $\mathrm{Fe}$ (III) ions were assayed by total organic carbon analyzer (TOC; Shimadzu TOC- $\mathrm{V}_{\mathrm{CHS}}$ ) and inductively coupled plasma atomic emission spectroscopy (ICP-AES; SII SPS 3520UV-2) measurements, respectively. The specific surface area of the hematite particles was investigated by $\mathrm{N}_{2}$ adsorption isotherms recorded by a computer-aided volumetric apparatus built-in-house at a boiling point of liquid- $\mathrm{N}_{2}$. Prior to the adsorption, the samples were treated at $100^{\circ} \mathrm{C}$ under $1.0 \times 10^{-3} \mathrm{~Pa}$ for $2 \mathrm{~h}$.

\subsection{Photocatalysis measurement}

The photocatalytic properties of as-synthesized hematite particles were evaluated by using Rhodamine $B(R B)$ used as a probe molecules as reported by Fang et al. ${ }^{20)}$ A suspension of hematite phocatalysts $(3 \mathrm{~mL}, 5 \mathrm{mg} / \mathrm{L})$ after dispersed by an ultrasonic agitation was added to a quartz cell $(1.0 \mathrm{~cm} \times 1.0 \mathrm{~cm} \times$ $4.5 \mathrm{~cm})$. The RB solution $(50 \mu \mathrm{L}, 1.5 \mathrm{mM})$ was injected into the quartz cell using a micropipet with continuous stirring under UV light irradiation of $365 \mathrm{~nm}(30 \mathrm{~W})$. The degree of RB degradation was monitored by absorption at a wavelength of $533 \mathrm{~nm}$ using a Halogen light source (UV-vis: Hitachi Hi-Tech U-3900H) for 5-20 min intervals. Since this measurement takes only a few second, no variation of solution temperature was encountered. All the measurements were carried out at $25^{\circ} \mathrm{C}$.

\section{Results and discussion}

\subsection{Morphology and crystal structure}

Figure 2 displays the TEM pictures of typical hematite particles precipitated by aging for $7 \mathrm{~d}$ at $0.8 \mathrm{wt} . \%$ of PEOs (L-11, R-400 and E-60) together with those produced without PEO (referred to control system). L-11 and R-400 produced pseudocubic particles though E-60 gave nearly spherical with large particle size distribution especially at high E-60 concentration. It is noteworthy that the fairly uniform pseudocubic particles can be seen for L-11 with low $M_{w}$ value and narrow molecular weight distribution.

Since the PEOs are nonionic polymers, there is no large difference can be seen among the solution pHs. All the system exhibit $\mathrm{pH}$ drop from ca. 1.8-1.9 to $1.0-1.1$ by hydrolysis of $\mathrm{FeCl}_{3}$ molecules. The mean edge length and/or particle diameter $\left(\mathrm{L}_{\mathrm{p}}\right)$ for the L-11, R-150, $-400,-1000$ and E-60 are displayed in Fig. 3(a). The $L_{p}$ values of all the systems remarkably dropped 


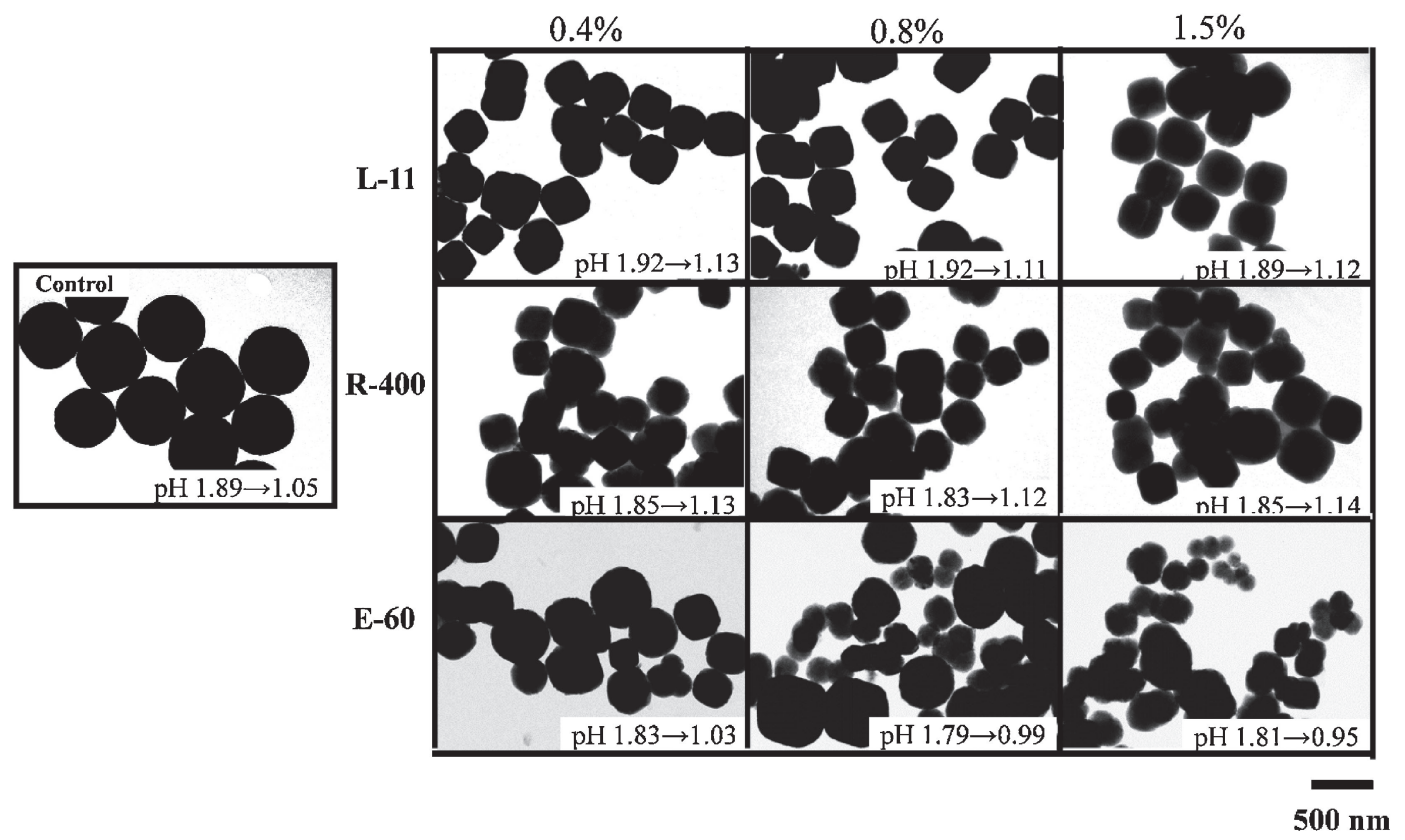

Fig. 2. TEM micrographs of typical hematite particles produced without PEO (control system) and with various amounts of L-11, R- -400 and E- 60 by aging at $100^{\circ} \mathrm{C}$ for $7 \mathrm{~d}$.
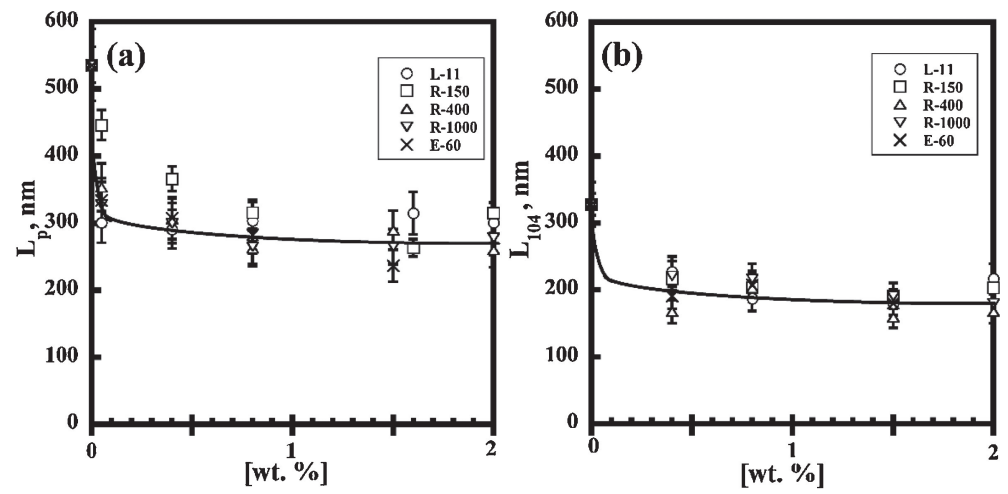

Fig. 3. (a) Mean edge length and/or particle diameter $\left(\mathrm{L}_{\mathrm{p}}\right)$ and (b) crystallite sizes of the particles $\left(\mathrm{L}_{104}\right)$ of hematite particles produced with various amounts of PEO by aging at $100^{\circ} \mathrm{C}$ for $7 \mathrm{~d}$.

from 540 to $300 \mathrm{~nm}$ after addition of $0.05 \%$ PEOs but their reduction was less up to $2 \mathrm{wt} . \%$ PEO ( $\mathrm{L}_{\mathrm{p}}$ is ca. $260 \mathrm{~nm}$ ). There is no large difference among five kinds of PEO molecules can be seen in $\mathrm{L}_{\mathrm{p}}$ values despite there are large difference in particle size distribution for these systems. To make clear the effect of PEOs onto the particle size distribution of particles produced, particle size distribution curves were made by counting over 300 particles for the systems produced with $0.8 \mathrm{wt} \%$ PEO in Fig. 4. The median, mode, number mean diameter, length mean diameter and standard deviation are listed in a table in Fig. 4 (bottom). It is easy to recognize that particle size distribution curves of $\mathrm{L}-11$ is sharp but they are spread out by increase in $\mathrm{M}_{\mathrm{w}}$ of PEOs $(\mathrm{R}-150<\mathrm{R}-400<\mathrm{R}-1000 \ll \mathrm{E}-60)$. This result indicated that the uniformity in size of pseudocubic hematite particles strongly depends on the molecular weight distribution of PEO; the nearly monodispersed pseudocubic hematite particles can be precipitated by using L-11 with narrow molecular size distribution. It can be presumed from above results that monodispersed pseudocubic hematite particles with $260 \mathrm{~nm}$ in diameter can be produced if we can acquire the PEO molecules with single molecular weight of $1 \times 10^{5}$.
Figure 5 displays the XRD patterns of the particles precipitated with L-11 and R-150 providing pseudocubic particles. Clearly, all the particles exhibit characteristics of hematite (JCPDS33-664). Similar results were obtained for the other PEO systems. However, the normally observed strong peak of the $\{012\}$ face $\left(2 \theta=24.2^{\circ}\right)$ becomes weak by increase in the concentrations of L-11 and R-150 systems. Hence, the relative area intensity of the $\{012\}$ peak was calculated and plotted as a function of concentrations of PEOs (abbreviated as [PEO]) in Fig. 6. Here the area intensity of the control system was assumed as unity. The relative area intensities are decreased for all the systems. This decrease in the $\{012\}$ peak corresponds to the results of Surfonamine system, ${ }^{19)}$ i.e., the pseudocubic crystal habit is thought to be formed by the specific adsorption of PEO molecules together with chloride ions and/or choloro ferric complexes to the $\{012\}$ faces, restraining the growth in the directions normal to the $\{012\}$ face. ${ }^{21)}$

The crystallite sizes of the particles $\left(\mathrm{L}_{104}\right)$ were evaluated using the Scherrer equation from the half height width of XRD peak of $\{104\}$ face $\left(2 \theta=33.2^{\circ}\right)$. The results are shown in Fig. 3(b). Clearly, the crystallite sizes are decreased with increase in the 

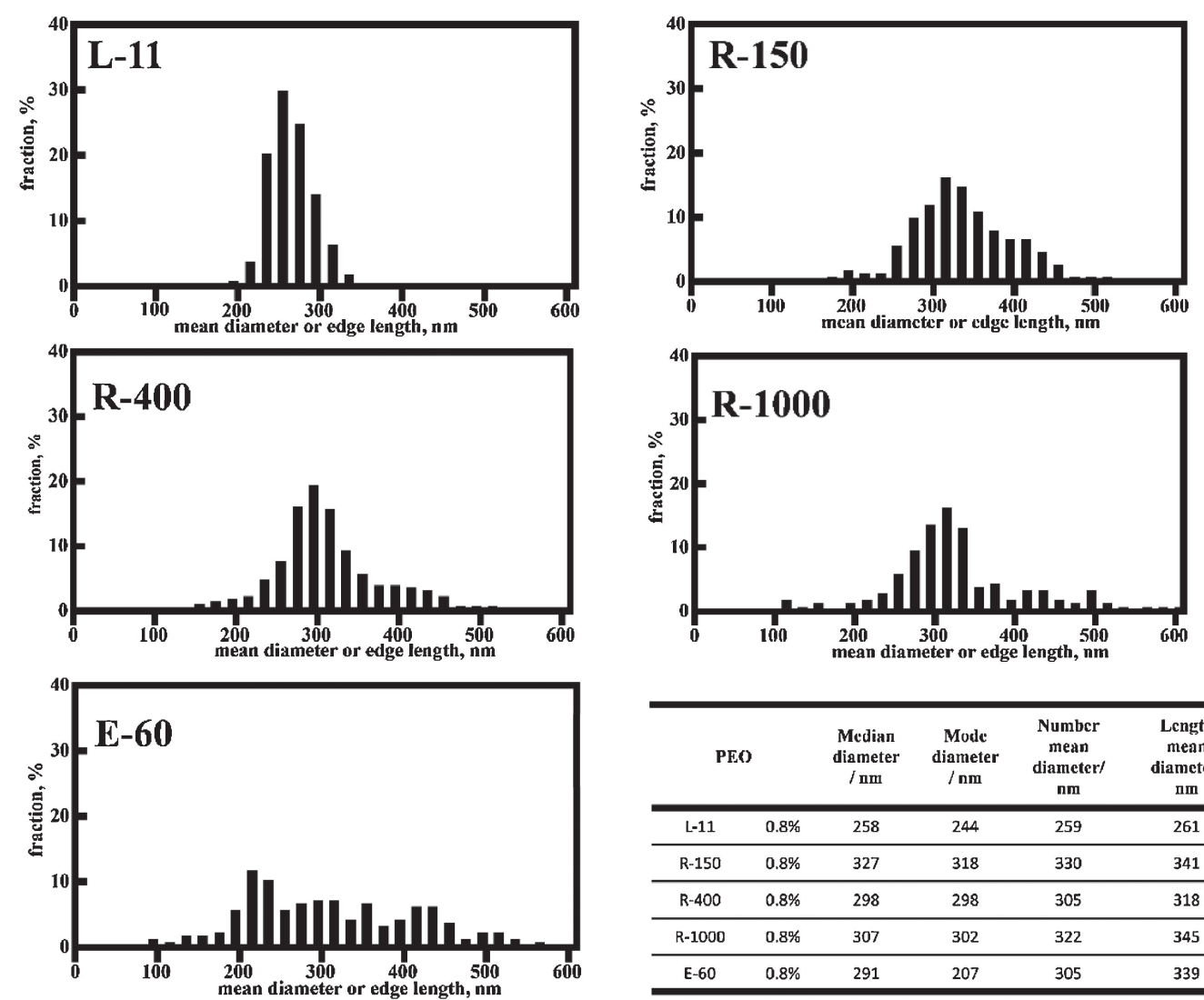

\begin{tabular}{ccccccc}
\hline PEO & $\begin{array}{c}\text { Median } \\
\text { diameter } \\
/ \text { nm }\end{array}$ & $\begin{array}{c}\text { Mode } \\
\text { diameter } \\
/ \mathbf{n m}\end{array}$ & $\begin{array}{c}\text { Numbcr } \\
\text { mean } \\
\text { diameter/ } \\
\text { nm }\end{array}$ & $\begin{array}{c}\text { Length } \\
\text { mean } \\
\text { diameter/ } \\
\text { nm }\end{array}$ & $\begin{array}{c}\text { Standard } \\
\text { deviation }\end{array}$ \\
\hline L-11 & $\mathbf{0 . 8 \%}$ & 258 & 244 & 259 & 261 & 26 \\
\hline R-150 & $0.8 \%$ & 327 & 318 & 330 & 341 & 60 \\
\hline R-400 & $0.8 \%$ & 298 & 298 & 305 & 318 & 63 \\
\hline R-1000 & $0.8 \%$ & 307 & 302 & 322 & 345 & 85 \\
\hline E-60 & $0.8 \%$ & 291 & 207 & 305 & 339 & 103 \\
\hline
\end{tabular}

Fig. 4. Particle size distribution curves of hematite particles produced with five kinds of PEO $(0.8$ wt.\%) and their mathematical analysis results of particle size.
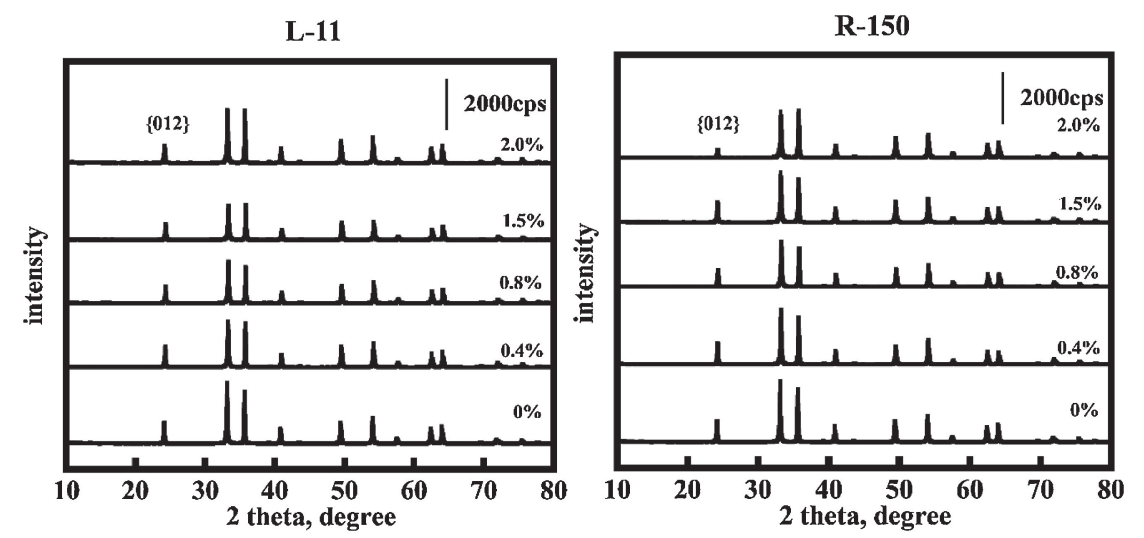

Fig. 5. XRD patterns of typical hematite particles produced with various amounts of L-11 and R-150 by aging at $100^{\circ} \mathrm{C}$ for $7 \mathrm{~d}$.

PEO concentration in each system and $\mathrm{L}_{104}$ are smaller than $\mathrm{L}_{\mathrm{p}}$ of the corresponding particles in Fig. 3(a), indicating that all the particles can be regarded as a polycrystal.

The TG-DTA curves of the particles produced with PEOs are shown in Fig. 7. The curves contain two mass loss steps around at $25-600^{\circ} \mathrm{C}$ and $600-1000^{\circ} \mathrm{C}$. Clearly the former mass loss is increased by raising the [PEO]. This mass loss can be ascribed to dehydration and dehydroxylation from the particle surface. The mass loss step at $600-1000^{\circ} \mathrm{C}$ is derived by elimination of $\mathrm{OH}^{-}$ions included in the hydrohematite particle. In hematite, $\mathrm{O}^{2-}$ may be partly replaced by $\mathrm{OH}^{-}$ions accompanying $\mathrm{Fe}$ (III) vacancies to provide charge balance of the particles. These anion-substituted hematites are called as hydrohematite or protohematite by Wolska and Schwertmann. ${ }^{22)}$ The TG-DTA curves of the particles produced with E-60 only exhibit a large weight loss at $200-400^{\circ} \mathrm{C}$ together with an exothermic peaks, indicating that the E-60 molecules remained in these particles. This result coincides with the result of TOC as will be described later.

\subsection{Specific surface area}

The adsorption isotherms of $\mathrm{N}_{2}$ for all the samples produced were shown the type II isotherms in the IUPAC classification. ${ }^{23)}$ The specific surface areas of the particles (SSA) were estimated by fitting the adsorption isotherms of $\mathrm{N}_{2}$ to the BET equation and plotted as a function of [PEO] in Fig. 8. All the SSA values are 


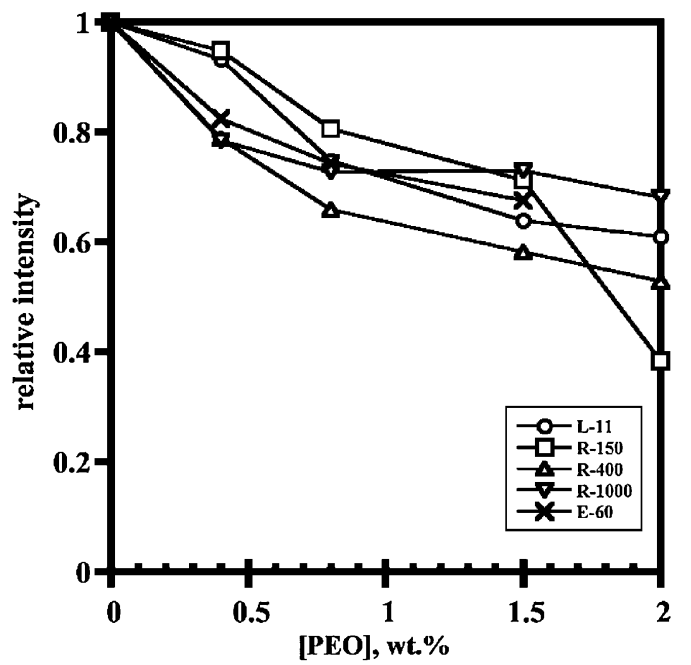

Fig. 6. Plots of relative intensity of $\{012\}$ face of XRD pattern as a function of PEO concentration ([PEO]) for five kinds of PEOs.

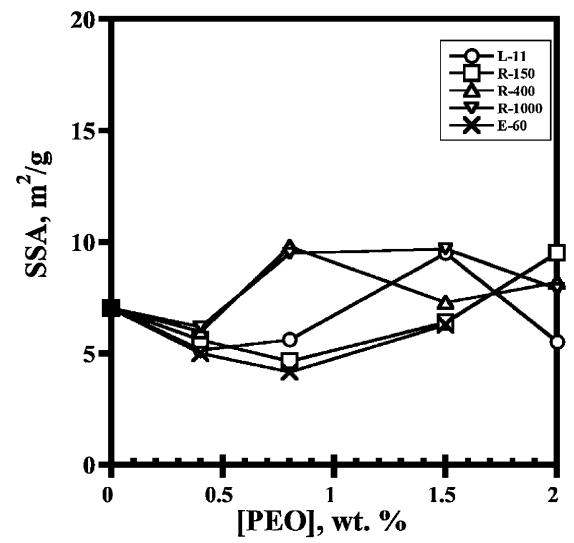

Fig. 8. Plots of specific surface area (SSA) of the hematite particles as a function of PEO concentration ([PEO]) for five kinds of PEOs.

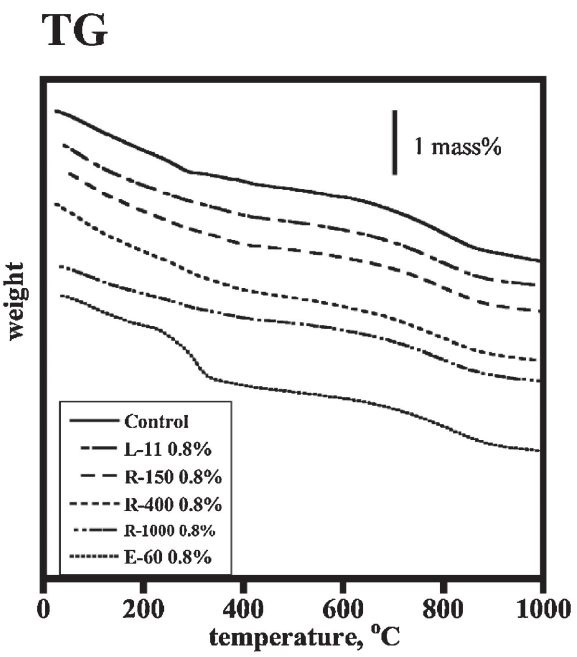

\section{DTA}

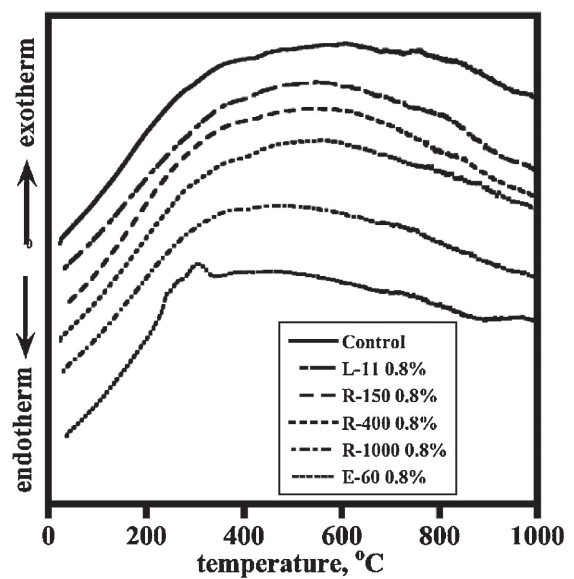

Fig. 7. TG-DTA curves of hematite particles produced with five kinds of PEO (0.8 wt. \%).

ranged in $4-10 \mathrm{~m}^{2} / \mathrm{g}$ and no large difference can be recognized. By assuming monodispersed cubic particles with $\mathrm{L}_{\mathrm{p}}=300 \mathrm{~nm}$ with $5.2 \mathrm{~g} / \mathrm{cm}^{3}$ density, SSA value can be calculated as $3.8 \mathrm{~m}^{2} / \mathrm{g}$. The measured SSA values are almost corresponded to the calculated SSA one, indicating that the pseudocubic hematite particles are non-porous. In the case of Surfonamine, the molecules remained between ultrafine PN particles adsorbing chloride ions and/or chloro ferric complexes on their surfaces and produced highly uniform micropores with diameters of $0.7 \mathrm{~nm} .{ }^{19)}$ This difference between Surfonamine and PEO employed in the present study can be attributed to the difference in adsorption ability of those polymers, i.e., adsorption ability of PEO is lower, especially at high temperature, than Surfonamine with terminated amino groups even though they have large molecular weight.

\subsection{Formation mechanism of pseudocubic hema- tite particles in the presence of PEO}

The authors reported that pseudocubic shape appers to be characteristic to hematite particles prepared in solution containing high concentration of chloride ions $(>0.1 \mathrm{M}) .{ }^{24)}$ We revealed that the dramatic shift of the isoelectric point from about 8.5 to 3.0 due to irreversible incorporation of chloride ions in the surface layers of pseudocubic hematite particles by a forced hydrolysis reaction though electrokinetic and XPS measurements after thoroughly washing the particles with alkaline solution. The pseudocubic crystal habit is thought to be formed by the specific adsorption of chloride ions and/or chloro ferric complexes to the $\{012\}$ faces, retaining the growth in the direction of normal to the $\{012\}$ faces as described before. Similar mechanism was also reported by a forced hydrolysis reaction of condensed ferric hydroxide gel, so called as gel-sol technique. ${ }^{21)}$ Hence it can be considered that the pseudocubic hematite particles were formed by the specific adsorption of PEO molecules together with chloride ions and/or chloro ferric complexes to the $\{012\}$ faces. The preparation of pseudocubic hematite particles from diluted alcoholic solutions of ferric chloride was also reported by Hamada and Matijević. ${ }^{25), 26)}$ They concluded that alcohol acts as an impurity, destroying the normal interfacial structure and providing an easier transition from solution to crystal thus affecting the growth rate.

To disclose the mechanism of the formation of pseudocubic hematite particles in the present study, we traced on the systems 
with 0.8 wt.\% of PEOs. In this experiment, we measured the time resolved TEM pictures together with the concentrations of Fe(III) ions (abbreviated as [Fe(III)]) and PEO (abbreviated as [PEO]) in a supernatant at different maturation time by TEM, ICP-AES and TOC, respectively. The hematite fraction in the precipitates was obtained and the results were shown in Fig. 9. Here the area intensity of fundamental vibration band of hematite at $580 \mathrm{~cm}^{-1}$ produced after maturation for $7 \mathrm{~d}$ was defined as unity in each system. Clearly, the control system (O) slowly produced spherical particles and the phase transformation from $\beta$-FeOOH to hematite was almost completed after aged for $5 \mathrm{~d}$ as well as reported in the previous paper. ${ }^{18)}$ However, the addition of PEOs enhanced the phase transformation, i.e., the phase transformation from tiny rod-like $\beta$-FeOOH to pseudocubic hematite particles was completed after aging for $2 \mathrm{~d}$, indicating that these PEO molecules enhance the particle growth. This is due to the formation of fine $\beta$-FeOOH particles, i.e., these particles are readily dissolved and transferred to hematite phase though the TEM pictures are not shown.

The changes of [Fe(III)] and [PEO] as a function of aging time were shown in Figs. 10(a) and 10(b), respectively. In the case for the control system in Fig. 10(a), [Fe(III)] decreases very rapidly within $6 \mathrm{~h}$ after started aging and slightly increases up to $12 \mathrm{~h}$ due to the dissolution of $\beta$-FeOOH particles $(\bigcirc)$. After aging for $1 \mathrm{~d},[\mathrm{Fe}(\mathrm{III})]$ decreases again and reaches a constant value (ca. $5 \mathrm{mmol} / \mathrm{dm}^{3}$ ), but it reduces again remarkably after aging over $5 \mathrm{~d}$. This rapid reduction of [Fe(III)] (secondary step) coincides with the phase transformation of $\beta$-FeOOH to hematite as is confirmed in Fig. 9. This agreement between secondary reduc-

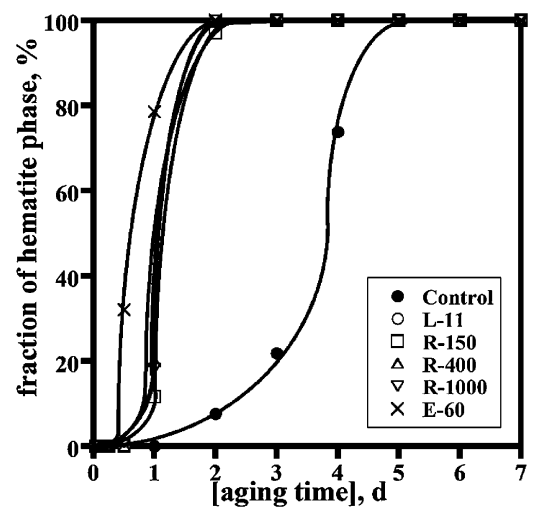

Fig. 9. Changes of fraction of hematite phase as a function of aging time on the five kinds of PEO $(0.8 \mathrm{wt} . \%)$ systems. tion step of $[\mathrm{Fe}(\mathrm{III})]$ and completion of phase transformation may indicate that the dissolution of $\beta$-FeOOH particle is a ratedetermining step in the reaction as the authors reported in a previous paper. ${ }^{18)}$ On the other hand, [Fe(III)] values for the systems with PEOs within $12 \mathrm{~h}$ are lower than the control system, indicating that the hydrolysis reaction of $\mathrm{Fe}(\mathrm{III})$ ions are accelerated at the initial stage of reaction. This acceleration may be attributed to the transfer of hydrated water from Fe(III) ions to PEO molecules in order to promote PEO dissolution at high temperature. The $[\mathrm{Fe}(\mathrm{III})]$ values after $12 \mathrm{~h}$, except for E-60 system (X), decreased steeply rather than the control system. The [Fe(III)] values for the L-11 ( $\square$ ) became to zero at $4 \mathrm{~d}$, suggesting a fast phase transformation from $\beta$ - $\mathrm{FeOOH}$ to hematite. This fast phase transformation may induce a monodispersed pseudocubic particles. On the contrary, the E-60 system showed a slow phase transformation exhibiting the high [Fe(III)] value. This extremely slow phase transformation is caused by the adsorption onto $\beta$ $\mathrm{FeOOH}$ and $\mathrm{PN}$ particles to inhibit both their dissolution and migration at $100^{\circ} \mathrm{C}$. According to this slow phase transformation, the polydispersed hematite particles could be precipitated. The amounts of [PEO] curves in Fig. 10(b) indicates that PEO molecules, except for E-60, do not adsorbed on $\beta$-FeOOH particles and pseudocubic hematite ones because these systems exhibited no change in [PEO]. The PEO molecules adsorbed on $\beta$ - $\mathrm{FeOOH}, \mathrm{PN}$ and hematite particles during aging at $100^{\circ} \mathrm{C}$ by reducing their solubility, but PEO molecules could be readily desorbed by reducing the aging solution to room temperature and completely removed after filtration. On the other hand, only the [PEO] for E-60 was steeply decreased to one-third within $1 \mathrm{~d}$. The desorption of E-60 with largest molecular weight was hardly to take place and remained on the hematite particle surface. The residual E-60 molecules was already confirmed by the TG-DTA curves as was shown in Fig. 7. From above results, it can be regarded the PEOs molecules, especially for the L-11, acted as a morphology control agent without remaining in/on the resulting particles.

\subsection{Photocatalitic function}

Fang et al. fabricated spherical hematite colloidal monocrystal cluster (CNCs) ${ }^{20)}$ The hematite CNCs have $2 \mu \mathrm{m}$ in diameter of secondary spherical particles and they are composed of numerous nanocrystals of approximately $20 \mathrm{~nm}$ in diameter. Since CMCs are aggregates of small nanocrystals, the CMCs have large SSA values of $36 \mathrm{~m}^{2} / \mathrm{g}$. Fang et al. reported that CMCs exhibit a high photocatalytic activity, i.e., almost $100 \%$ of RB was decomposed within $1 \mathrm{~h}$ by UV light irradiation. This high photocatalytic
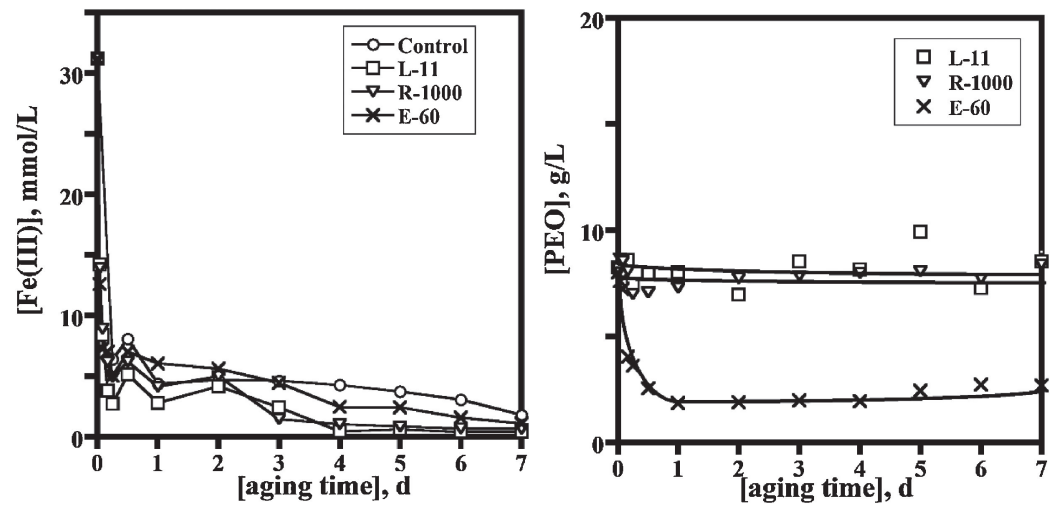

Fig. 10. Plots of (a) $[\mathrm{Fe}(\mathrm{III})]$ and (b) [PEO] as a function of aging time on the control and three kinds of PEO (0.8 wt.\%: L-11. R-1000 and E-60) systems. 


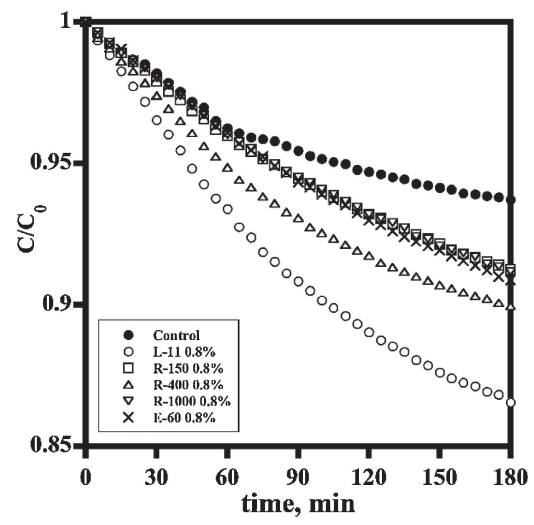

Fig. 11. Degradation rate of UV absorption of RB over different hematite particles produced without PEO (control) and with $0.8 \mathrm{wt} . \%$ five kinds of PEOs.

activity is due to their high SSA. Hence, it is interesting to examine whether the pseudocubic hematite particles possess photocatalytic activity or not.

Figure 11 displays the degradation curves of RB by using different kinds of hematite particles produced with PEO in the present study as photocatalysts. Prior to this experiment, we confirmed that no depression of RB concentration was taken place by adsorption onto hematite particles under dark condition. The large spherical particles produced at the control system exhibit a photocatalytic nature, i.e., ca. $5 \%$ of RB was decomposed within $3 \mathrm{~h}(\mathbf{O})$. Slightly fast degradation of $\mathrm{RB}$ can be seen in pseudocubic particles produced with R-150, $-400,-1000$ and E-60. This improvement of photocatalytic activity may be due to their large size with flat crystal surfaces of pseudocubic particles. Indeed, the monodispersed pseudocubic particles produced with L-11 exhibit the highest photocatalystic activity. This result indicates that the photocatalytic reaction is strongly depend on the size and monodispersity of the pseudocubic hematite particles with flat surface. However, the photocatalytic activity is lower than CMCs reported by Fang et al. ${ }^{20)}$ because of their low SSA values. It is necessary to study the effect of surface properties on the photocatalytic activity of hematite particles in future.

\section{Conclusion}

The monodispersity of pseudocubic hematite particles was controlled by using five kinds of PEO with various molecular weights and distribution. The fairly uniform pseudocubic particles was precipitated for PEO with low $\mathrm{M}_{\mathrm{w}}$ value and narrow molecular weight distribution. However, POEs with large molecular weight and wide molecular weight distribution produced polydispersed pseudocubic hematite particles. Moreover, the PEO with the largest molecular weight with wide molecular weight distribution attained nearly spherical with large particle size distribution. All the pseudocubic hematite particles were grown by aggregation of polynuclear $(\mathrm{PN})$ primary particles and there were regarded as a polycrystal. The $\mathrm{N}_{2}$ adsorption measurement indicated that the pseudocubic hematite particles are non-porous. The pseudocubic crystal habit was formed by the specific adsorption of PEO molecules together with chloride ions and/or chloro ferric complexes to the $\{012\}$ faces, restraining the growth process through stacking of ultrafine PN particles in the direction of normal to the $\{012\}$ faces but strictly restricting the growth and mutual fusion of PN ones. The uniform pseudocubic particles exhibit effective photocatalytic behavior own to their large size with uniform flat crystal faces.

Acknowledgements The authors are thankful to Mr. Yasuhiro Isono and Mr. Kunio Tada, Meisei Chemical Works Ltd. Kyoto, Japan for kindly supplying Alkox ${ }^{\circledR}$.

\section{References}

1) Y. Sun and Y. Xia, Science, 298, 2176-2179 (2002).

2) Z. A. Peng and X. Peng, J. Am. Chem. Soc., 124, 3343-3353 (2002).

3) X. Wang, J. Zhuang, Q. Peng and Y. Li, Nature, 437, 121-124 (2005).

4) Y. Yin and A. P. Alivisatos, Nature, 437, 664-670 (2005).

5) U. Schwertmann and R. M. Cornell, Iron Oxide, Structure, Properties, Reactions, Occurrence and Uses, VCH (1996).

6) L. S. Zhong, J. S. Hu, H. P. Liang, A. M. Cao, W. G. Song and L. J. Wan, Adv. Mater., 18, 2426-2431 (2006).

7) S. Y. Zeng, K. B. Tang, T. W. Li, Z. H. Liang, D. Wang, Y. K. Wang and W. W. Zhou, J. Phys. Chem. C, 111, 10217-10225 (2007).

8) Y. Piao, J. Kim, H. B. Na, D. Kim, J. S. Baek, M. K. Ko, J. H. Lee, M. Shokouhimehr and T. Hyeon, Nat. Mater., 7, 242-247 (2008).

9) X. Wen, S. Wang, Y. Ding, Z. L. Wang and S. Yang, J. Phys. Chem. B, 109, 215-220 (2005).

10) C. J. Jia, L. D. Sun, Z. G. Yan, L. P. You, F. Luo, X. D. Han, Y. C. Pang, Z. Zhang and C. H. Yan, Angew. Chem., Int. Ed., 44, 4328-4333 (2005).

11) L. H. Liu, H. Z. Kou, W. Mo, H. Liu and Y. Wang, J. Phys Chem. B, 110, 15218-15223 (2006).

12) S. B. Wang, Y. L. Min and S. Y. Yu, J. Phys. Chem. C, 111, 3351-3354 (2007)

13) X. Liang, X. Wang, J. Zhuang, Y. Chen, D. Wang and Y. Li, Adv. Funct. Mater., 16, 1805-1813 (2006).

14) K. Kandori, IRON OXIDE PARTICLES, in "Encyclopedia of Surface and Colloid Science, Second Edition", Ed. by Auther Hubbert, Taylor \& Francis: New York (2006) pp. 3126-3138.

15) J. K. Bailey, C. J. Brinke and M. L. Mecartney, J. Colloid Interface Sci., 157, 1-13 (1993).

16) K. Kandori, N. Okamoto and T. Ishikawa, Langmuir, 18, 2895-2900 (2002).

17) K. Kandori, N. Yamamoto, A. Yasukawa and T. Ishikawa, Phys. Chem. Chem. Phys., 4, 6116-6122 (2002).

18) K. Kandori, Y. Yamoto and T. Ishikawa, J. Colloid Interface Sci., 283, 432-439 (2005).

19) K. Kandori and M. Watanabe, Colloid Polym. Sci., 289, 981991 (2011).

20) X. L. Fang, C. Chen, M. S. Jin, Q. Kuang, Z. X. Xie, S. Y. Xie, R. B. Huang and L. S. Zheng, J. Mater. Chem., 19, 6154-6160 (2009).

21) T. Sugimoto, A. Muramatsu, K. Sakata and D. Shindo, J. Colloid Interface Sci., 158, 420-428 (1993).

22) E. Wolska and U. Schwertmann, Z Kristallogr, 189, 223-238 (1989).

23) K. S. W. Sing, D. H. Everett, R. A. W. Haul, L. Moscou, L. Pierotti, R. A. Rouquerol and J. Siemieniewska, Pure Appl. Chem., 57, 603-620 (1985).

24) K. Kandori, Y. Kawashima and T. Ishikawa, J. Mater. Sci. Lett., 12, 288-290 (1993).

25) S. Hamada and E. Matijević, J. Colloid Interface Sci., 84, 274277 (1981).

26) S. Hamada and E. Matijević, J. Chem. Soc., Faraday Trans. I, 78, 2147-2154 (1982). 